\title{
Polyol Profiles in Down Syndrome myo-Inositol, Specifically, Is Elevated in the Cerebrospinal Fluid
}

\author{
H. Umesha Shetty, Mark B. Schapiro, Harold W. Holloway, and Stanley I. Rapoport \\ Laboratory of Neurosciences, National Institute on Aging, National Institutes of Health, Bethesda, Maryland 20892
}

\begin{abstract}
Polyols are reduction products of aldoses and ketoses; their concentrations in tissues can reflect carbohydrate metabolism. Several polyol species were quantitated in cerebrospinal fluid (CSF) and plasma from 10 Down Syndrome (trisomy 21 ) subjects between the ages of 22 and 63 years $(3$ of whom were demented) and from 10 healthy age-matched controls, using a gas chromatographic/mass spectrometric technique. The mean CSF concentration and the mean CSF/ plasma concentration ratio of $m y o$-inositol were significantly elevated in Down syndrome compared with controls, but were not correlated with the presence of dementia in the Down subjects. Plasma myo-inositol was not significantly altered in these subjects. No significant difference between Down syndrome and controls was found for CSF concentrations of mannitol, sorbitol, galactitol, ribitol, arabitol, or 1,5anhydrosorbitol, but plasma mannitol, ribitol and arabitol were elevated in Down syndrome. The present observation provides new impetus for studying synthesis and transport of myo-inositol as well as phosphatidylinositol cycle in trisomy 21 disorder. (J. Clin. Invest. 1995. 95:542-546.) Key words: carbohydrates - phosphatidylinositols • brain • trisomy $\cdot$ Alzheimer disease
\end{abstract}

\section{Introduction}

The Down syndrome (DS) ${ }^{1}$ (trisomy 21 ) phenotype is characterized by a multitude of morphological and functional abnormalities, including short stature, microcephaly, cardiovascular malformations, immunological disorders, leukemia, and cataracts. In addition, mental retardation and, after the fourth decade of life, neuropathological and neurochemical changes similar to those observed in Alzheimer disease are the universal manifestations of this genetic anomaly $(1,2)$. At the cellular level, altered electrical properties, decreased $\mathrm{Na}^{+} / \mathrm{K}^{+}$-ATPase activity and exaggerated response to certain drugs point to dysfunction of the plasma membrane in DS.

In DS, gene products of the extra chromosome 21 may cause abnormal levels of certain metabolites (by synthesis, transport,

Address correspondence to $\mathrm{H}$. Umesha Shetty, Ph.D., Laboratory of Neurosciences, National Institute on Aging, National Institutes of Health, Building 10, Room 6C 103, Bethesda, MD 20892. Phone: 301496-8970; FAX: 301-402-0074. 1994

Received for publication 6 July 1994 and in revised form 7 October

1. Abbreviations used in this paper: CSF, cerebrospinal fluid; DS, Down syndrome; $R_{C S F}$, cerebrospinal fluid to plasma concentration ratio.

The Journal of Clinical Investigation, Inc.

Volume 95, February 1995, 542-546 catabolism, and regulatory pathways) directly or by affecting gene expression of other chromosomes and subsequent molecular events. We thus sought to identify metabolites whose altered levels in brain might contribute to pathophysiology relevant to DS. Polyols, the ubiquitous metabolites of sugars, play a significant role in diabetic neuropathy (3), uremic polyneuropathy (4), and galactosemia (5). In these metabolic disorders, decreased $\mathrm{Na}^{+} / \mathrm{K}^{+}$-ATPase, altered electrical properties and other plasma membrane abnormalities are associated with elevated tissue polyols. Additionally, cataract formation is a major pathological consequence of abnormal metabolism of polyols (6). These pathophysiologic abnormalities reminiscent of certain DS phenotypes guided us in an examination of polyols in DS subjects.

Here we report the results of quantitative profiling of polyols in cerebrospinal fluid (CSF) and plasma from DS adults and age-matched healthy controls. As polyols are reduction products of sugars, analysis of polyol levels in DS may be used to probe glycolytic, pentose phosphate and the connected pathways for abnormalities related to this genetic disorder.

\section{Methods}

Selection of subjects. All DS subjects had trisomy 21 except for one demented older subject who had a mosaic translocation, mos46, $\mathrm{xx} /$ $46, x x,-21,+(21 q ; 21 q)(7)$. DS subjects and healthy volunteers were carefully screened according to procedures previously described (8). The subjects underwent a review of medical history and a physical and neurological examination (blood and urine tests, electrocardiograph, chest radiograph, electroencephalograph, and structural brain imaging). One young DS subject had a mild depression. The control subjects had no history of neurological disorder; these subjects and the selected DS subjects were free of medical conditions ( such as hypertension, seizures, diabetes, malignancy, and trauma) that might have affected the central nervous system. Except for thyroid replacement in several DS subjects, no DS or control subject was taking medication. No subject had a history of alcohol or drug abuse. The DS group consisted of 10 adults, 5 males, and 5 females, with ages ranging from 22 to $63(43.3 \pm 15.1$, mean \pm SD) years. Four of these were classified as young DS subjects with ages ranging from 22 to 34 (27.2 \pm 4.9 , mean $\pm S D)$ years. Ages of remaining six older DS subjects ranged from 45 to 64 ( $54.0 \pm 7.3$, mean \pm SD) years. Three of the older DS subjects were found by examination, history, and cognitive testing to be demented $(9,10)$. Subsequently, 2 of the other 3 older DS subjects became demented. The control group consisted of 10 subjects, 7 males, and 3 females, with ages ranging from 23 to 69 $(47.5 \pm 17.1$, mean $\pm S D)$ years. No control subject was demented. The research was conducted under National Institutes of Health protocols $81 \mathrm{AG} 10$ (for subjects with DS) and 80AG26 (for controls).

Sample collection. All subjects were free of medication for at least 2 wk before lumbar puncture and were placed on a diet that was low in monoaminergic precursors for $72 \mathrm{~h}$ (11). The morning after overnight bed rest and fasting, lumbar punctures were performed with subjects in the lateral decubitus position. After a sterile preparation, the L3-4 interspace was infiltrated with $1 \%$ lidocaine. A 20 -gauge spinal needle then was inserted into the spinal subarachnoid space. Specimens were analyzed only if the CSF was clear, with normal cell counts, protein, 
and glucose. The first $12 \mathrm{ml}$ of CSF were pooled and immediately placed on wet ice. One-ml aliquots then were frozen at $-80^{\circ} \mathrm{C}$ until assayed. Just before the lumbar puncture, venous blood was withdrawn through an indwelling intravenous catheter placed $10 \mathrm{~h}$ before (to minimize acute changes of blood and CSF neurotransmitters). Stable blood pressures and heart rates were recorded over three or more successive 10-min periods before venous sampling. These measurements were made in order to assess the level of stress, which could in turn influence the concentrations of some neurotransmitters. The blood was placed in heparinized polypropylene tubes and immediately centrifuged at 5,000 $g$ at $4^{\circ} \mathrm{C}$ for $20 \mathrm{~min}$. One-ml aliquots of the resultant plasma were removed and then stored at $-80^{\circ} \mathrm{C}$ until assayed.

Gas chromatographic/mass spectrometric assay of polyols. Polyol species in CSF and plasma were quantitated by a method developed in our laboratory (Shetty, H. U., H. W. Holloway, and S. I. Rapoport, manuscript submitted for publication). Briefly, CSF $(25 \mu \mathrm{l})$ or plasma $(100 \mu \mathrm{l})$ was mixed with internal standard $\left(\left[{ }^{2} \mathrm{H}_{6}\right]\right.$ myo-inositol $)$, deproteinized and evaporated under vacuum. The residue was heated with acetic anhydride/pyridine/4-dimethylaminopyridine, then dissolved in hexane/ethyl acetate and washed with sodium bicarbonate solution. The organic layer was evaporated to dryness, and the residue was reconstituted in ethyl acetate. An aliquot of this solution was injected into the gas chromatograph/mass spectrometer. Polyols were resolved on a capillary column (bonded $50 \%$ phenyl-50\% methyl polysiloxane), and individual species were detected and quantitated by a chemical ionization technique in an ion trap mass spectrometer (12). Each polyol yielded a highly abundant fragment ion corresponding to the loss of one $\mathrm{CH}_{3} \mathrm{COOH}$ residue from the protonated molecule. The ions, $\mathrm{m} / \mathrm{z} 273$ for 1,5-anhydrosorbitol, $\mathrm{m} / \mathrm{z} 303$ for ribitol, arabitol and xylitol (stereoisomers), $\mathrm{m} / \mathrm{z} 373$ for $m y o$-inositol, and $\mathrm{m} / \mathrm{z} 375$ for mannitol, sorbitol and galactitol (stereoisomers) were monitored. The constituent ion $\mathrm{m} / z 379$ of the internal standard was also acquired simultaneously. The concentration of each polyol species in CSF or plasma was read from the standard curve generated. The relative standard deviation for quantitation was not $>8 \%$ for CSF polyols and $15 \%$ for plasma polyols.

Materials. Polyol species were obtained from Sigma Chemical Co. (St. Louis, MO). Deuterium-labeled internal standard was from Merck Sharp \& Dohme/Isotopes (Canada). Pyridine and acetic anhydride were obtained from Alltech Associates, Inc. (Deerfield, IL), and solvents were from Burdick \& Jackson (Muskegon, MI). The gas chromatograph/mass spectrometer was a Finnigan MAT ITS40 ${ }^{\mathrm{TM}}$ (San Jose, CA). The capillary column was from Restek Corporation (Bellefonte, PA).

Statistical analysis. The mean concentration of each polyol in CSF or plasma of the DS group was compared with the respective mean of the control group by a one-tailed $t$ test, and the results were corrected for multiple comparisons by a Bonferroni procedure (13). The CSF to plasma concentration ratio $\left(R_{C S F}\right)$ was calculated for each subject, and the mean values were similarly compared. Also, the difference in variances was checked by an $F$ test, and the results were corrected for multiple comparisons. The statistical difference between mean $R_{C S F}$ and a hypothesized mean 1.0 was determined by a one-sample $t$ test. A correlation analysis on variables was performed by linear regression. The criterion for statistical significance was $P<0.05$.

\section{Results}

The gas chromatographic/mass spectrometric technique enabled quantitation of the polyol species, ribitol, arabitol, 1,5-anhydrosorbitol, myo-inositol, mannitol, sorbitol, and galactitol in CSF and plasma and, additionally, xylitol in plasma. Xylitol was barely detectable in CSF. A representative ion chromatogram for CSF polyols from a DS subject is shown in Fig. 1. The chromatographic retention time and mass of the ion generated for each polyol and the internal standard, $\left[{ }^{2} \mathrm{H}_{6}\right]$ myo-inositol, are shown. In the ion chromatogram for plasma samples (not shown) the peak for xylitol was distinct, allowing reliable quantitation of the polyol. There was no gross difference between the ion chromatograms of control and DS subjects. Only the peak area ratio of polyol to internal standard was altered for certain polyols in DS.

CSF polyol concentrations for healthy control and DS subjects are shown in Table I. Mean concentrations of ribitol, arabitol, 1,5-anhydrosorbitol, mannitol, sorbitol and galactitol in DS were not significantly different from those of control subjects. As with control subjects, the CSF concentration of xylitol was negligibly low in DS. The CSF level of myo-inositol, $36.32 \pm 8.02 \mathrm{ng} / \mu \mathrm{l}$ (mean $\pm \mathrm{SD})$, in DS was significantly $(P$ $<0.005$ ) higher (by $49 \%$ ) than $24.35 \pm 4.20 \mathrm{ng} / \mu \mathrm{l}$ found in control subjects. In each group of 10 subjects, the CSF myoinositol concentration ranged from 27.17 to $56.03 \mathrm{ng} / \mu \mathrm{l}$ for DS and from 15.76 to $32.02 \mathrm{ng} / \mu$ l for control subjects. These data as well as corresponding $R_{C S F}$ values are illustrated in Fig. 2.

Within the DS group, mean myo-inositol concentration was found to be $37.56 \pm 10.00 \mathrm{ng} / \mu \mathrm{l}$ for the 6 old subjects, which was not significantly different $(P>0.05)$ from $34.45 \pm 4.26$ $\mathrm{ng} / \mu \mathrm{l}$ found in the four young subjects. The mean concentration in three demented old DS subjects was $34.53 \pm 6.43 \mathrm{ng} / \mu \mathrm{l}$. In the subject with a mosaic translocation, the myo-inositol level was $27.17 \mathrm{ng} / \mu \mathrm{l}$. In the entire group of $10 \mathrm{DS}$ subjects, no correlation ( $r=0.33, P>0.05)$ was observed between height and CSF myo-inositol concentration. Whereas the age of subject and the level of myo-inositol correlated in the control group ( $r$ $=0.73, P<0.05)$, no such correlation was found in the DS group.

Plasma polyol concentrations for DS and control subjects are shown in Table II. Three polyols were found significantly elevated in DS: mannitol (by 113\%), ribitol (by 34\%) and arabitol (by $66 \%$ ). Also, there was greater variability ( $P$ $<0.001$ ) in the concentration of mannitol and arabitol in DS than in controls. The mean plasma level of myo-inositol in DS did not differ $(P>0.05)$ from the control value, in contrast to the elevated mean level in CSF.

Calculated mean CSF to plasma concentration ratios, $R_{C S F}$, for the polyols in DS and in control subjects are shown in Table III. The values were $>1.0$ for all polyols except $1,5-$ anhydrosorbitol $(P>0.05)$. Corresponding to their elevated plasma levels, $R_{C S F}$ values for ribitol and arabitol were significantly lower in DS compared with controls. No significant reduction in this ratio for mannitol was observed. Because of its significant elevation in CSF but not in plasma, the calculated $R_{C S F}$ for myo-inositol was significantly higher (31\%) in DS than in controls. CSF and plasma levels correlated for 1,5anhydrosorbitol in the control group $(r=0.70, P=0.01)$ as well as in DS group $(r=0.76, P=0.01)$ and also for myoinositol in the control group ( $r=0.70, P<0.05$ ) only. Correlation between the levels in these two compartments was not found for other polyols.

\section{Discussion}

Polyols are ubiquitous metabolites formed by the reduction of aldoses and ketoses. The sugar precursors of polyols are essential substrates of glycolytic and pentose phosphate pathways. If these metabolic pathways were affected by a disease, the metabolic imbalance could manifest itself as altered concentration profiles of polyols. Abnormal carbohydrate metabolism in disorders such as diabetic neuropathy, galactosemia and essential 


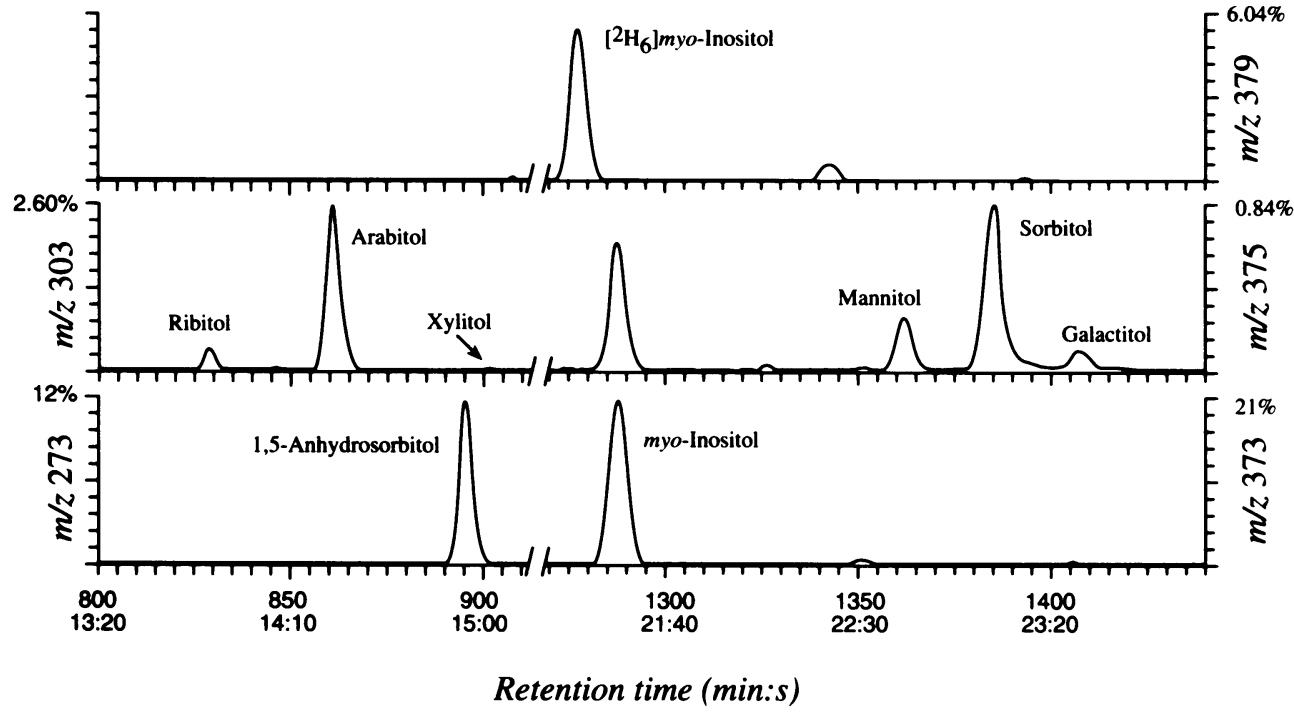

Figure 1. Ion chromatogram generated by the gas chromatographic/mass spectrometric analysis of the acetate derivatives of polyols in CSF from a DS subject. pentosuria leads to a large deviation in the polyol levels in the central nervous system and peripheral organs $(5,14,15)$. The data presented here show severalfold higher concentrations of mannitol, sorbitol, galactitol, ribitol, arabitol, and myo-inositol in CSF than plasma. CSF levels of these polyols, with the exception of myo-inositol in the control group, do not correlate with plasma levels, both in control and DS subjects. However, 1,5-anhydrosorbitol with an $R_{C S F}$ value close to 1.0 shows a strong correlation between its levels in two compartments. These disposition characteristics indicate that CSF polyols, except 1,5-anhydrosorbitol, are mostly of brain origin $(16,17)$. Therefore, levels of polyols in CSF likely reflect the general status of metabolism of sugar precursors, transport systems and other regulatory mechanisms related to brain.

myo-Inositol, specifically in CSF, is elevated in DS adults. The $R_{C S F}$ value is also higher, reflecting elevated brain levels in DS. The turnover of myo-inositol in the central nervous system is not fully understood. myo-Inositol is synthesized within brain from glucose (18); it also can enter brain from blood using a low capacity, saturable transport system at the bloodbrain barrier (19). Animal experiments indicate that a smaller percentage (20\% in rabbits and $2 \%$ in rats) of myo-inositol in CSF is derived from blood and the remainder from the brain $(20,21)$. It appears that both de novo synthesis in brain and transport from blood regulate the slowly turning over myo-

Table I. CSF Polyol Concentrations for Control and DS Subjects

\begin{tabular}{lcc}
\hline \multicolumn{1}{c}{ Polyols in CSF } & $\begin{array}{c}\text { Control subjects }(n=10) \\
\text { mean } \pm \text { SD }(\mathrm{ng} / \mu \mathrm{l})\end{array}$ & $\begin{array}{c}\text { DS subjects }(n=10) \\
\text { mean } \pm \mathrm{SD}(\mathrm{ng} / \mu \mathrm{l})\end{array}$ \\
\hline Mannitol & $0.872 \pm 0.248$ & $0.947 \pm 0.622$ \\
Sorbitol & $2.51 \pm 0.61$ & $2.55 \pm 1.54$ \\
Galactitol & $0.343 \pm 0.125$ & $0.326 \pm 0.172$ \\
Ribitol & $0.580 \pm 0.108$ & $0.535 \pm 0.136$ \\
Arabitol & $3.68 \pm 0.95$ & $3.33 \pm 0.90$ \\
1,5-Anhydrosorbitol & $15.40 \pm 3.75$ & $18.13 \pm 5.25$ \\
myo-Inositol & $24.35 \pm 4.20$ & $36.32 \pm 8.02 *$ \\
& & \\
\hline
\end{tabular}

$* P<0.005$. inositol pool of the brain. Thus in DS, a higher level of cerebral myo-inositol is achieved, probably either by increased synthesis or by the transport system. Peripherally, DS fibroblasts show a two- to threefold increased uptake of myo-inositol, yet the cells have normal levels of myo-inositol, suggesting increased turnover $(22,23)$. DS brain cells have not been investigated for their uptake properties. myo-Inositol is principally catabolized in the kidney (24); however, this process is unlikely to be impaired in DS since the plasma level is unaffected.

Trisomy 21 leads to Alzheimer disease generally after the fourth decade of life. However, levels of elevated CSF myoinositol found in young adult DS subjects were comparable with levels in the DS subjects over 40 years of age, indicating that the abnormality is not secondary to the neurodegenerative Alzheimer process. The quantitative data also indicate that elevation of CSF myo-inositol is unrelated to dementia. Additionally, it appears that shorter stature in DS, which might affect where along the neuraxis the CSF is sampled, has no bearing on the

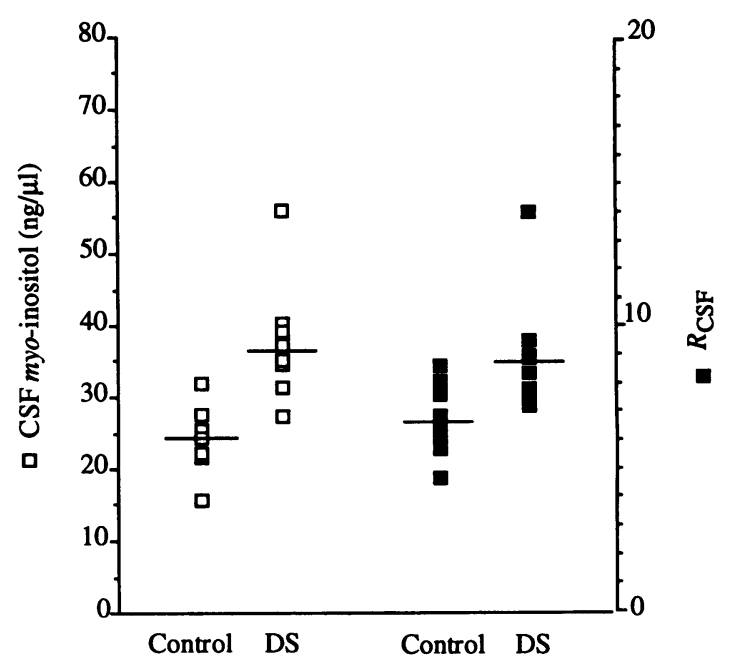

Figure 2. Scatter plot of CSF myo-inositol concentrations (open squares) and CSF to plasma concentration ratios, $R_{C S F}$ (closed squares), in control and DS subjects. Means are indicated by horizontal bars. 
Table II. Plasma Polyol Concentrations for Control and DS Subjects

\begin{tabular}{lcc}
\hline \multicolumn{1}{c}{ Polyols in plasma } & $\begin{array}{c}\text { Control subjects }(n=10) \\
\text { mean } \pm \text { SD }(\mathrm{ng} / \mu \mathrm{l})\end{array}$ & $\begin{array}{c}\text { DS subjects }(n=10) \\
\text { mean } \pm \text { SD }(\mathrm{ng} / \mu \mathrm{l})\end{array}$ \\
\hline Mannitol & $0.268 \pm 0.036$ & $0.572 \pm 0.250^{*}$ \\
Sorbitol & $0.193 \pm 0.071$ & $0.156 \pm 0.076$ \\
Galactitol & $0.067 \pm 0.024$ & $0.105 \pm 0.057$ \\
Ribitol & $0.071 \pm 0.014$ & $0.095 \pm 0.021 \ddagger$ \\
Arabitol & $0.388 \pm 0.053$ & $0.646 \pm 0.278 \ddagger$ \\
Xylitol & $0.102 \pm 0.017$ & $0.120 \pm 0.037$ \\
1,5-Anhydrosorbitol & $15.41 \pm 5.21$ & $22.23 \pm 9.82$ \\
myo-Inositol & $3.78 \pm 1.01$ & $4.22 \pm 0.60$ \\
& & \\
\hline
\end{tabular}

${ }^{*} P<0.005 ; \quad{ }^{\ddagger} P<0.05$.

increased CSF levels of myo-inositol because all other polyols are at normal levels. Also in the present study no correlation was detected between levels of CSF myo-inositol and the heights of DS subjects. In other studies in DS adults, rostrocaudal gradients of choline did not differ in DS versus controls (11).

Regardless of the mechanism involved, the elevated cerebral myo-inositol could considerably affect and be related to disturbed cellular functions such as membrane turnover, signal transduction and osmoregulation. The membrane phospholipid, phosphatidylinositol is synthesized from myo-inositol and CDPdiacylglycerol by the enzyme CDP-1,2-diacyl-sn-glycerol:myoinositol 3-phosphatidyltransferase. The purified enzyme from rat brain has been reported to exhibit a $K_{m}$ of $4.6 \mathrm{mM}$ for myoinositol (25). The normal level of myo-inositol in neurons is near this $K_{m}$ value (26). Apparently, an increased level of myoinositol can lead to an augmented synthesis of phosphatidylinositols, including the phosphorylated form, phosphatidylinositol4,5 -biphosphate $(27,28)$. The reported increased incorporation of myo-inositol into phospholipids in DS fibroblasts argues for such a possibility (22). Thus in DS, an increased turnover of inositol phospholipids could exert a profound effect on phosphatidylinositol cycle dynamics when cells are stimulated by an agonist. Additionally, altered turnover of brain phosphatidylcholine in DS adults is suggested by the finding of elevated CSF, though not plasma, choline (11). Together, these findings argue for increased membrane instability in DS.

myo-Inositol is also a cerebral osmolyte (29), but the effect of its elevation on the structural and functional integrity of the central nervous system is not known. However, dorsal root ganglion cells (culture, mouse embryo) revealed ultrastructural abnormalities when exposed to 109 to $1,090 \mu \mathrm{M}$ myo-inositol (30). In humans, uremic polyneuropathy is associated with elevated plasma myo-inositol. When this condition was mimicked in rats, a reduction in sciatic motor-nerve conduction velocity was observed (4).

Polyols such as sorbitol, galactitol and xylitol have been implicated in the development of sugar cataracts (6). Although myo-inositol is maintained in the lens of the eye at a high level by an intraocular transport system (31), its physiological role in the lens is not clear. However, a defect in the metabolism of myo-inositol may affect osmoregulatory and, perhaps, other functions in the lens. Thus in DS the lenses may be subjected to physiological stress if myo-inositol's profile in this compartment is also altered. The metabolic basis for a high incident of
Table III. The CSF to Plasma Concentration Ratios, $R_{C S F}$, for Polyols

\begin{tabular}{lcc}
\hline \multicolumn{1}{c}{ Polyols } & $\begin{array}{c}\text { Control subjects }(n=10) \\
R_{C S F}(\text { mean } \pm \text { SD })\end{array}$ & $\begin{array}{c}\text { DS subjects }(n=10) \\
R_{C S F}(\text { mean } \pm \text { SD) }\end{array}$ \\
\hline Mannitol & $3.34 \pm 1.12$ & $1.97 \pm 1.66$ \\
Sorbitol & $15.20 \pm 7.53$ & $21.02 \pm 16.84$ \\
Galactitol & $5.75 \pm 3.00$ & $3.89 \pm 2.49$ \\
Ribitol & $8.23 \pm 1.60$ & $5.96 \pm 2.14^{*}$ \\
Arabitol & $9.69 \pm 2.97$ & $5.97 \pm 2.66^{*}$ \\
Xylitol & $\ll 1.00$ & $\ll 1.00$ \\
1,5-Anhydrosorbitol & $1.04 \pm 0.201$ & $0.88 \pm 0.21$ \\
myo-Inositol & $6.65 \pm 1.21$ & $8.71 \pm 2.05^{*}$ \\
\hline
\end{tabular}

${ }^{*} P<0.05$.

cataracts and other ocular abnormalities in DS is not understood, although the gene for alpha- $\mathrm{A}_{2}$ subunit of crystallin has been localized to the trisomy segment of chromosome 21 (32). However, the potential involvement of myo-inositol in metabolic eye diseases cannot be overlooked.

CSF levels of mannitol, sorbitol, galactitol, ribitol, arabitol, xylitol, and 1,5-anhydrosorbitol are not altered in DS, indicating that the metabolism of sugars from which these polyols are derived is unimpaired in the central nervous system. Also, brain glucose metabolism in DS, as measured with positron emission tomography and $\left[{ }^{18} \mathrm{~F}\right] 2$-fluoro-2-deoxy-D-glucose, is similar to metabolism in healthy subjects (33). Thus, brain glycolytic and pentose phosphate pathways may not be affected in DS and a generalized alteration of these pathways is not the cause of increased CSF myo-inositol. Furthermore, no elevation in CSF polyols except for myo-inositol and $R_{C S F}>2.0$ for most polyols were found, indicating that the brain-CSF barrier or plasma-CSF barrier for these sugar metabolites is not functionally different in this genetic disorder.

Dietary input directly influences the plasma level of myoinositol (24). The plasma level is unchanged in DS, indicating that absorption, synthesis and catabolism processes for myoinositol are unaffected in peripheral organs. Other sugar metabolites-mannitol, sorbitol, galactitol, ribitol, arabitol, xylitol and 1,5-anhydrosorbitol-are linked to glycolytic and pentose phosphate pathways. Increased plasma levels of mannitol, ribitol and arabitol in DS suggest some aberration in carbohydrate metabolism of peripheral organs. Certain enzymes of the glycolytic and pentose phosphate pathways are increased in erythrocytes from DS (34), which may account for the elevated metabolites. In addition, the gene coding the phosphofructokinase (liver type) has been localized to chromosome 21 (32). Overexpression of the gene encoding this regulatory enzyme of the glycolytic pathway could lead to altered levels of sugar metabolites.

A 1.5-time increase of CSF myo-inositol in DS may be the result of a global disregulation in metabolism or of increased dosage of a single gene on chromosome 21 . An investigation into the myo-inositol and phosphatidylinositol synthetase enzymes as well as properties of the myo-inositol transport and uptake systems may allow one to elucidate the mechanism and, possibly, facilitate identification of the responsible gene. Finally, the effect of altered myo-inositol homeostasis on the development of brain and its role in the pathogenesis of DS, 
mental retardation and Alzheimer disease in particular, need to be addressed.

\section{Acknowledgments}

We thank Drs. K. Pettigrew and K. Hatanpaa for valuable suggestions on statistical analysis of the data.

\section{References}

1. Epstein, C. J. 1986. Trisomy 21 and the nervous system: from cause to cure. In The Neurobiology of Down Syndrome. C. J. Epstein, editor. Raven Press, New York. 1-15.

2. Schapiro, M. B., J. V. Haxby, and C. L. Grady. 1992. Nature of mental retardation and dementia in Down syndrome: study with PET, CT, and neuropsychology. Neurobiol. Aging. 13:723-734.

3. Cohen, M. P. 1987. The Polyol Paradigm and Complications of Diabetes Springer-Verlag, New York. 59 pp.

4. Clements, R. S., Jr., P. V. DeJesus, Jr., and A. I. Winegrad. 1973. Raised plasma-myoinositol levels in uraemia and experimental neuropathy. Lancet. i:1137-1141.

5. Egan, T. J., and W. W. Wells. 1966. Alternate metabolic pathway in galactosemia. Am. J. Dis. Child. 111:400-405.

6. Kinoshita, J. H. 1974. Mechanisms initiating cataract formation. Invest. Ophthalmol. 13:713-724.

7. Schapiro, M. B., A. Kumar, B. White, D. Fox, C. L. Grady, J. V. Haxby, R. P. Friedland, and S. I. Rapoport. 1990. Dementia without mental retardation in mosaic translocation Down syndrome. Brain Dysfunct. 3:165-174.

8. Duara, R., R. A. Margolin, E. A. Robertson-Tchabo, E. D. London, M Schwartz, J. W. Renfrew, B. J. Koziarz, M. Sundaram, C. Grady, A. M. Moore D. H. Ingvar, L. Sokoloff, H. Weingartner, R. M. Kessler, R. G. Manning, M. A Channing, N. R. Cutler, and S. I. Rapoport. 1983. Cerebral glucose utilization, as measured with positron emission tomography in 21 resting healthy men between the ages of 21 and 83 years. Brain. 106:761-775.

9. Haxby, J. V. 1989. Neuropsychological evaluation of adults with Down's syndrome: patterns of selective impairment in non-demented old adults. J. Ment. Defic. Res. 33:193-210.

10. Schapiro, M. B., J. V. Haxby, C. L. Grady, R. Duara, N. L. Schlageter, B. White, A. Moore, M. Sundaram, S. M. Larson, and S. I. Rapoport. 1987. Decline in cerebral glucose utilization and cognitive function with aging in Down's syndrome. J. Neurol. Neurosurg. Psychiatry. 50:766-774.

11. Schapiro, M. B., J. R. Atack, I. Hanin, C. May, J. V. Haxby, and S. I. Rapoport. 1990. Lumbar cerebrospinal fluid choline in healthy aging and in Down's syndrome. Arch. Neurol. 47:977-980.

12. Shetty, H. U., E. M. Daly, N. H. Greig, S. I. Rapoport, and T. T. Soncrant. 1991. An automatic reaction control chemical ionization technique in ion trap detector for quantitative plasma profiling of arecoline in treated Alzheimer patients. J. Am. Soc. Mass Spectrom. 2:168-173.

13. Shaffer, J. P. 1988. Simultaneous testing. In Encyclopedia of Statistical Sciences, Volume 8. S. Kotz and N. L. Johnson, editors. John Wiley \& Sons, New York. 484-490.
14. Servo, C., L. Bergstrom, and R. Fogelholm. 1977. Cerebrospinal fluid sorbitol and myoinositol in diabetic polyneuropathy. Acta Med. Scand. 202:301304

15. Touster, O., and D. R. D. Shaw. 1962. Biochemistry of the acyclic polyols Physiol. Rev. 42:181-225.

16. Rapoport, S. I. 1983. Passage of proteins from blood to cerebrospinal fluid. In Neurobiology of Cerebrospinal Fluid, Volume 2. J. H. Wood, editor. Plenum Press, New York. 233-245.

17. Davson, H., K. Welch, and M. B. Segal. 1987. The Physiology and Pathophysiology of the Cerebrospinal Fluid. Churchill Livingstone, New York $247 \mathrm{pp}$.

18. Hauser, G., and V. N. Finelli. 1963. The biosynthesis of free and phosphatide myo-inositol from glucose by mammalian tissue slices. J. Biol. Chem 238:3224-3228.

19. Spector, R. 1988. Myo-inositol transport through the blood-brain barrier Neurochem. Res. 13:785-787.

20. Spector, R., and A. V. Lorenzo. 1975. Myo-inositol transport in the centra nervous system. Am. J. Physiol. 228:1510-1518.

21. Barkai, A. I. 1981. Myo-inositol turnover in the intact rat brain: increased production after $d$-amphetamine. J. Neurochem. 36:1485-1491.

22. Fruen, B. R., and B. R. Lester. 1990. Down's syndrome fibroblasts exhibit enhanced inositol uptake. Biochem. J. 270:119-123.

23. Fruen, B. R., and B. R. Lester. 1991. Inositol and inositol 1,4,5-triphosphate content of Down syndrome fibroblasts exhibiting enhanced inositol uptake. FEBS (Fed. Eur. Biochem. Soc.) Lett. 295:43-47.

24. Holub, B. J. 1986. Metabolism and function of myo-inositol and inositol phospholipids. Annu. Rev. Nutr. 6:563-597.

25. Ghalayini, A., and J. Eichberg. 1985. Purification of phosphatidylinositol synthetase from rat brain by CDP-diacylglycerol affinity chromatography and properties of the purified enzyme. J. Neurochem. 44:175-182.

26. Berridge, M. J., C. P. Downes, and M. R. Hanley. 1989. Neural and developmental actions of lithium: a unifying hypothesis. Cell. 59:411-419.

27. Yagi, K., and A. Kotaki. 1969. The effect of massive doses of myo-inositol on hepatic phospholipid metabolism. Ann. NY Acad. Sci. 165:710-725.

28. Nahorski, S. R., C. I. Ragan, and R. A. John Challiss. 1991. Lithium and the phosphoinositide cycle: an example of uncompetitive inhibition and its pharmacological consequences. Trends Pharmacol. Sci. 12:297-303.

29. Heilig, C. W. M. E. Stromski, J. D. Blumenfeld, J. P. Lee, and S. R. Gullans. 1989. Characterization of the major brain osmolytes that accumulate in salt-loaded rats. Am. J. Physiol. (Renal Fluid Electrolyte Physiol. 26). 257:F1108-1116.

30. Liveson, J. A., J. Gardner, and M. B. Bornstein. 1977. Tissue culture studies of possible uremic neurotoxins: Myo-inositol. Kidney Int. 12:131-136.

31. Varma, S. D., B. Chakrapani, and V. N. Reddy. 1970. Intraocular transport of myoinositol II. Accumulation in the rabbit lens in vitro. Invest. Ophthalmol. 9:794-800.

32. Tippett, P., and J.-C. Kaplan. 1985. Report of the committee on the genetic constitution of chromosomes 20, 21, and 22. Cytogenet. Cell Genet. 40:268-295.

33. Schapiro, M. B., C. L. Grady, A. Kumar, P. Herscovitch, J. V. Haxby, A. M. Moore, B. White, R. P. Friedland, and S. I. Rapoport. 1990. Regional cerebral glucose metabolism is normal in young adults with Down syndrome. $J$. Cereb. Blood Flow Metab. 10:199-206.

34. Hsia, D. Y.-Y., P. Justice, G. F. Smith, and R. M. Dowben. 1971. Down's syndrome. A critical review of the biochemical and immunological data. Am. J. Dis. Child. 121:153-161. 